\title{
Technical aspects of uniportal video-assisted thoracoscopic sleeve resections: Where are the limits?
}

Diego González-Rivas, MD, FECTS, ${ }^{\text {a,b }}$ Alejandro Garcia, MD, ${ }^{\mathrm{b}}$ Chang Chen, MD, ${ }^{\mathrm{a}}$ Yang Yang, MD, ${ }^{\mathrm{a}}$ Yuming Zhu, MD, ${ }^{\mathrm{a}}$ and Gening Jiang, $\mathrm{MD}^{\mathrm{a}}$

Video clip is available online.

Feature Editor's Note-Global enthusiasm for a uniportal approach to video-assisted thoracoscopic (VATS) resection of thoracic neoplasms has seen a surge during the past decade subsequent to the first reported case in 2010. Proponents of the approach argue for diminished postoperative pain, reduced hospital length of stay, and more rapid recovery compared with a 3-hole VATS technique. Of note, in the only published randomized study that compared uniportal with other VATS techniques for lobectomy, there was no difference in postoperative outcomes including pain, length of stay, or complications (Perna et al. Eur J Cardiothorac Surg. 2016;50:411-5). Nevertheless, safety and feasibility of the technique are well established and the adoption of more advanced resections by champions of the modality are being explored as depicted in this technical report by GonzalezRivas and colleagues. Bronchial and/or vascular sleeve resections during pulmonary resection are challenging even in the presence of a thoracotomy, so achieving this operation through VATS or robotic assisted surgery and now uniportal VATS is noteworthy. This has been greatly facilitated by improved instrumentation and refinement of the uniportal technique that optimizes the parallel nature of visualization and surgical dissection. Importantly, this technique should only be implemented in the hands of surgeons familiar with the method in which appropriate oncologic resection can be achieved. As the authors emphasize incision placement is critical and many surgical pearls are

From the a Department of Thoracic Surgery, Shanghai Pulmonary Hospital, Tongji University School of Medicine, Shanghai, China; and ${ }^{\mathrm{b}}$ Department of Thoracic surgery and Minimally Invasive Thoracic Surgery Unit (UCTMI), Coruña University Hospital, Coruña, Spain.

Received for publication Dec 3, 2019; revisions received Dec 3, 2019; accepted for publication Feb 2, 2020; available ahead of print March 4, 2020.

Address for reprints: Diego González-Rivas, MD, FECTS, Department of Thoracic Surgery, Coruña Hospital, Xubias 84, 15006. Coruña, Spain (E-mail: diego. gonzalez.rivas@sergas.es).

JTCVS Techniques 2020;2:160-4

2666-2507

Copyright $@ 2020$ The Authors. Published by Elsevier Inc. on behalf of The American Association for Thoracic Surgery. This is an open access article under the CC BY-NC-

ND license (http://creativecommons.org/licenses/by-nc-nd/4.0/).

https://doi.org/10.1016/j.xjtc.2020.02.018

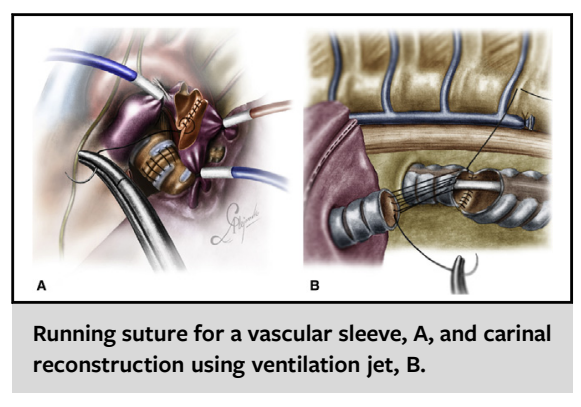

CENTRAL MESSAGE

A single running suture using 1 thread with 2 needles makes endto-end bronchovascular anastomosis faster and easier by minimally invasive thoracic surgery, especially with uniportal VATS.

See Commentaries on pages 165 and 167.

described in this report including avoidance of polymer clips for arterial vessel ligation and eliminating instruments that crowd the small access incision. Because sleeve resections require anastomoses of airway or vasculature, multiple sutures in an already crowded field is cumbersome. Conversion from the previous technique of interrupted suture on the cartilaginous wall and continuous running suture on the membranous wall to a single double-armed monofilament running suture to complete the entire 360 degrees of the anastomosis with a single knot appears to be a significant step forward. Among the many reported improvements to permit advanced pulmonary or carinal resection via a uniportal technique, the most important contribution of these authors is the dedication to teaching this technique to a wide spectrum of thoracic surgeons all over the world.

\section{Michael Lanuti, MD}

In recent years, experience gained through video-assisted thoracoscopic surgery (VATS), the development of better and adapted surgical instruments, and improvements in thoracoscopic cameras have greatly contributed to the ability to perform more challenging cases by VATS, 
such as parenchyma-sparing (sleeve) pulmonary resections. ${ }^{1,2}$

These procedures are recommended for the treatment of central tumors involving the origin of the lobar bronchus or extending into the main bronchus and in the case that the hilar lymph nodes are infiltrated, regardless of the patient's capacity to tolerate a pneumonectomy. Data accumulated over the past decades indicate that the same oncologic outcome can be achieved with these procedures but with lower morbidity, mortality, and improved postoperative quality of life, especially when done through VATS.

Traditionally, sleeve resections by VATS were considered a contraindication because of their technical difficulty by this approach. However, nowadays these procedures are performed by many thoracic VATS-experienced surgeons worldwide. ${ }^{3}$ Most surgeons use 3 to 4 incisions when a sleeve resection is needed. The uniportal technique, however, due to its geometric features and in expert hands, could facilitate this type of procedure, thus avoiding additional incisions. Currently, there are few articles showing results of uniportal VATS sleeve resections. We have recently published our experience with a significant number of cases at Shanghai Pulmonary Hospital, showing good outcomes. ${ }^{4}$

The bronchial and particularly the vascular sleeve resections with an end-to-end anastomosis are probably the most technically complex procedures in minimally invasive thoracic surgery. Therefore, the use of high-definition $30^{\circ}$ cameras and specific instruments with double-jointed articulation is required for these procedures.

In order of difficulty, we can divide the sleeve resections into 3 different groups: bronchial, vascular (single or double), and carinal and tracheal reconstructions.

\section{BRONCHIAL SLEEVE}

One of the most important requirements for uniportal sleeve resections is proper placement of the incision. It is advisable to use the fourth intercostal space, either for upper or lower sleeves in both the right and left sides to achieve a more comfortable instrumentation for suturing, especially for the deepest and most posterior areas aspects of the bronchus. A lower placement of the incision could make the anastomosis more difficult than necessary.

The right upper lobe bronchoplasty is normally the most frequently performed and least-difficult procedure due to the alignment of the main and the intermediate bronchi. However, because the right bronchus is located at the rear side of the pulmonary artery, it occasionally is too hidden by the pulmonary artery or the azygos vein, which increases the difficulty of the anastomosis. When the pulmonary artery interferes with the bronchial anastomosis, there are several maneuvers to keep it out of the way. It can be dissected and retracted laterally with a vessel loop or clamped and retracted with a tourniquet. For sleeve lobectomies, we do not recommend the use of polymer clips for small branches to avoid possible interference with the suture during the anastomosis.

On the right side, the bronchial mismatch is usually not a problem for the anastomosis except when we perform a right lower sleeve with the anastomosis from the middle lobe bronchus to the intermedius bronchus. This type of sleeve is usually performed due to tumors at the entrance of the inferior bronchus. The middle lobe bronchus must be cut tangentially to allow a greater bronchial surface for the anastomosis and thus reduce the caliber discrepancy.

In cases of sleeve bilobectomy, the key is to perform the anastomosis from a cranial view with caudal retraction of the upper lobe. Extra care must be taken to avoid rotation of the bronchus during suturing, making sure to align the membranous and cartilaginous portions correctly. To identify both parts of the bronchus and avoid malrotation, a very useful trick is to remember that the membranous portion runs parallel to the spine.

For a bronchial end-to-end anastomosis, different types of suture materials and techniques have been described in the literature, with all having shown to be reliable when the suture is tension free and the tissue is adequately vascularized. The decision to use a larger or smaller needle depends on personal preference. The use of a larger needle $(26 \mathrm{~mm})$ facilitates an easier rotation of the needle into the bronchus and allows suturing of both edges with a single movement.

For the first uniportal sleeve resection that our group described in the literature, the same principle as in open surgery was followed, that is, the use of interrupted sutures for the cartilaginous portion and a continuous suture for the membranous portion. However, the handling of many sutures for the interrupted portion by VATS was time consuming and not easy. ${ }^{5}$

To simplify the procedure, we switched to a doublearmed running suture (single thread with 2 needles). The idea is to perform a circumferential anastomosis of $360^{\circ}$ with both needles $\left(180^{\circ}\right.$ with each needle) and a single knot at the most anterior portion of the suture anastomosis. A single, monofilament, nonabsorbable, 30 double-armed suture for every bronchial sleeve anastomosis is normally used (a polydioxanone suture may be used as an alternative when available). This procedure is facilitated when dissection of the lymph node paratracheal and subcarinal stations are performed before the reconstruction. ${ }^{6}$

A continuous suture can reduce the time required for the completion of the anastomosis, not only because additional sutures are not required, but also because tangling is minimized when using a single suture. The first bite is placed "inside-out" at the far border between the cartilaginous and membranous part of the proximal bronchial tree and then connecting it "outside-in" to the distal bronchial 
tree, progressing in a counter-clockwise fashion incorporating the entire bronchial wall (full-thickness).

When performing the anastomosis, pulling both ends of the double-armed suture through the incision makes it is easy to get the suture tangled. A very good trick is to anchor one of the needles to the posterior pleura to keep that suture end pulled back and out of the way. Using the other needle, the lateral anastomosis is initiated and in this way interference with the opposite end of the double-armed suture is avoided.

Suturing is continued with the same needle, along the medial side of the circumference of the anastomosis, until at least half of the anastomosis has been completed.

The assistant surgeon helps during the suturing by applying the necessary tension to ensure a firm reapproximation of the proximal and distal bronchial structures and preventing the loosening of the suture. The second needle, which was anchored in the posterior pleura to keep it out of the way, is then used for the remaining half of the anastomosis circumference by starting "inside-out" at the distal bronchial structure and then connecting it "outside-in" at the proximal bronchial tree, progressing in a clockwise direction. Suturing is continued laterally until the first suture is met, completing the circumference. For the last 2 or 3 bites, the suture should be left relatively loose so that direct vision of the proximal and distal lumen can be maintained. This facilitates correct placement of the needle for the final bites. Before tying, a hook instrument is used to check the traction along the lateral side of the anastomosis for equal tension distribution. The distance from stitch to stitch should be no more than 2 to $2.5 \mathrm{~mm}$. The distance between the stitches should be adjusted if there is a caliber discrepancy between the proximal and distal bronchus, such as when the middle lobe is connected to the bronchus intermedius after a right lower sleeve lobectomy, or when the right upper lobe bronchus is connected to the carina following a tracheal sleeve bilobectomy. ${ }^{7}$

In uniportal VATS, the middle lobe sleeve is especially difficult. The location of the basal artery, and the instrumentation required to perform the anastomosis of the intermedius bronchus and basal bronchus from an anterior view, make this procedure challenging by a single anterior incision. For this reason, it is recommended to retract the pulmonary artery by using 2 tourniquets and to perform the anastomosis from posteriorly after anteriorly retracting the upper and lower lobe.

On the left side, sleeve resections are normally more difficult. The difficulty of left upper lobe (LUL) sleeve depends on the location of the main pulmonary artery in relation to the bronchus. If the artery is located posterior to the bronchus, the anastomosis could be easy due to the lateral alignment of the main and lower bronchus. However, if the artery location completely covers both bronchial stumps, the anastomosis could be very challenging.
Even in the easiest scenario, the artery should be always retracted out of the way for the posterior stitches. It can be encircled with a vessel loop and fixed to the posterior pleura, retracted with tourniquets, or retracted during every stitch with a long-curved suction.

The left lower sleeve lobectomy is technically more challenging than the LUL sleeve due to the orientation of the upper lobe bronchus to be reimplanted to the main bronchus from an anterior view position, as the main bronchus is usually located quite deep, below the descending aorta. Occasionally, when the origin of the lingular artery comes from the basal arterial trunk or when it complicates the bronchial dissection, one branch can be sacrificed to perform a more secure anastomosis.

In the case of endobronchial carcinoid tumors that are located purely inside the bronchus, a lung-sparing segmental bronchial resection can be performed. The bronchus intermedius resection is the easiest lung-sparing sleeve procedure. For tumors located purely on the main bronchus, the resection of the right side is less difficult compared with the left side despite the short size of right main bronchus. For the thoracoscopic reconstruction of a second carina on the left, the anastomosis is more complex because of the suturing of the upper and lower lobe bronchus from an anterior view, and the left main bronchus is often hidden behind the left pulmonary artery.

\section{VASCULAR OR DOUBLE SLEEVE}

The end-to-end anastomosis of the pulmonary artery is one the most technically difficult procedures by VATS. The most frequent location is the upper lobectomy on the left side. Double-sleeve resections on the right side are extremely rare and very challenging because of the deep location of the pulmonary artery stump for the anastomosis, as it is usually hidden by the superior cava vein.

In the initial vascular sleeve procedures we described in 2013, we used 2 vascular clamps through the incision, but this instrumentation was inconvenient as it took up unnecessary space in the incision. ${ }^{8}$ In 2014, we described the first uniportal double sleeve using a vascular clamp for the main pulmonary artery and a bulldog clamp for distal artery. ${ }^{9}$

After gaining experience with these procedures, we developed the use of tourniquets to clamp the artery both at the proximal and distal edges. To ensure correct and safe clamping of the artery, it is necessary to tie the tourniquet with silk and leave it inside the thoracic cavity. To avoid movement of the tourniquet, we use a wet gauze to keep it fixed in the apex and at the diaphragmatic face of the thoracic cavity (Video 1).

It is advisable to perform an intrapericardial dissection and dissect the pulmonary artery proximal to the ductus arteriosum to place the tourniquet in this position. In this way, the ductus acts as a stop to prevent accidental displacement (Figure 1, A). It should be taken into account that when the 


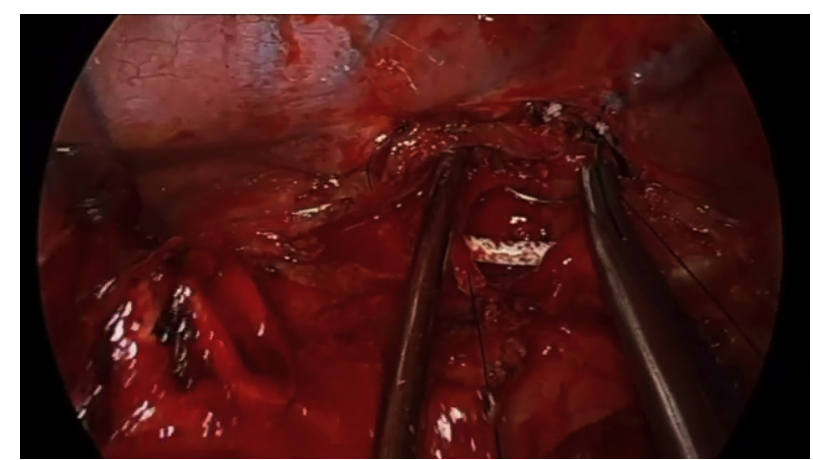

VIDEO 1. Uniportal VATS left upper sleeve lobectomy (0-05:31 minutes) and uniportal VATS RUL and carinal reconstruction (5:31-11:39 minutes). Video available at: https://www.jtcvs.org/article/S2666-2507(20)30124-3/ fulltext.

artery is cut that there is considerable proximal retraction. Therefore, it is important to not pull the lobe too much to avoid tearing the artery proximally and to always make the cut as distal as possible (when tumor allows). In the case of tumors with a very central and proximal location, the most advisable step is to use a clamp and divide the ductus (prolongs the length of the artery $0.8-1 \mathrm{~cm}$ ). The clamp should be placed outside the wound protector to decrease interference during the anastomosis.

For a vascular reconstruction following a vascular sleeve resection, the suturing technique we apply is the same as the one described for the bronchus (360 circumferential suture). A 4-0 or 5-0 double-armed PROLENE suture (needle size no more than $16 \mathrm{~mm}$ ) is preferred (Figure $1, A) .{ }^{5}$ It is recommended to irrigate both stumps of the artery with a heparin solution before starting the reconstruction to wash away debris from the endothelium and prevent subsequent pulmonary embolism. In the case of a bronchovascular sleeve resection, transection of the artery precedes that of the bronchus, whereas reconstruction of the bronchus precedes that of the artery to avoid tension on the vascular anastomosis. ${ }^{10}$

At the beginning of the procedure, it is advisable to perform a systematic aortopulmonary, paratracheal, and subcarinal lymphadenectomy, as well as release of the ligament or opening of the pericardium to reduce the time of arterial clamping. The bronchial anastomosis of LUL is the least challenging sleeve due to the lateral orientation of the bronchial stumps and the absence of the pulmonary artery. When the invasion of the pulmonary artery is very extensive, it is advisable to perform a bronchial sleeve even in the absence of endobronchial involvement to reduce the distance of the arterial anastomosis and, thus, reduce tension on the suture.

When the tumor extensively involves the fissure, dissection of the basal branch and A6 independently with 2 tourniquets is recommended to increase the surface of the distal arterial stump. In the event of finding difficulty in the dissection, the inferior pulmonary vein may be clamped instead of the distal artery.

\section{CARINAL AND TRACHEAL SLEEVE}

The placement of the incision for distal trachea or carinal resections should by the right side, fourth intercostal space. In case of tracheal resections in the lower part of trachea, an incision in the third intercostal space could also be used. A nonextensive pure tracheal resection at this location is not difficult because there are no structures interfering with the anastomosis.

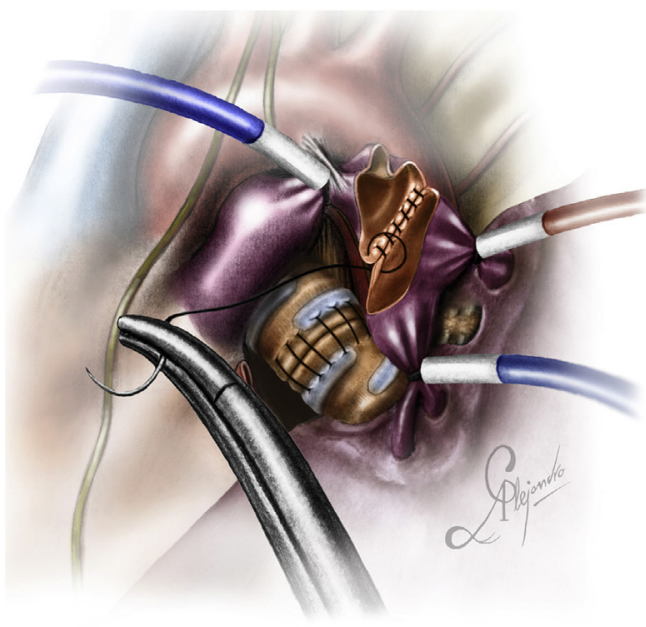

A

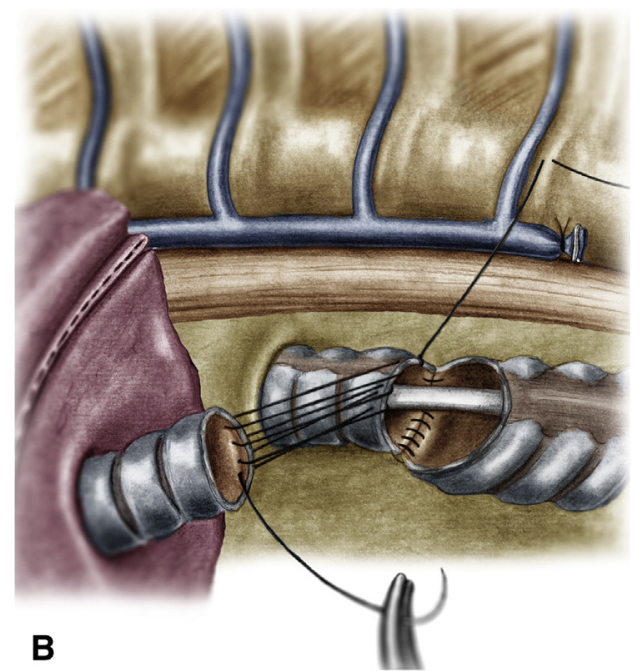

FIGURE 1. Illustration showing double sleeve and carinal reconstruction. A, Running suture for vascular sleeve anastomosis during a double-sleeve reconstruction. The tourniquets are placed proximal to the ductus to avoid an accidental displacement. B, Carinal reconstruction after right upper sleeve lobectomy combined with carinal resection using ventilation jet. 
To perform these procedures through a uniportal VATS, we recommend the use of a high-frequency ventilation jet to maintain lung ventilation. The catheter can be introduced through the endotracheal tube and thanks to the small diameter of the catheter for ventilation, it doesn't interfere with the initial part of the anastomosis.

When a right upper lobectomy is associated with carinal resection, the difficulty of the anastomosis could be increased. Meticulous reimplantation of the bronchus intermedius to the trachea or left mainstem bronchus without tension is crucial to avoid airway necrosis and narrowing. To avoid anastomotic angulation, devascularization, or excessive tension, it is recommended to perform maneuvers for hilar release before the anastomosis (release the ligament and U-shaped pericardial incision). The azygos vein must be transected and the stumps sutured laterally to the chest wall to improve the exposure of the field.

To avoid aspiration of blood into the left main bronchus, the circumferential tracheal or carinal incision should be performed without removing the double-lumen endotracheal tube. This maneuver allows a safe incision and resection of the distal trachea, right main and left main bronchus. The use of high-frequency jet ventilation of the left lung is useful to maintain oxygenation, and it facilitates the anastomosis of the left side wall of the left main stem bronchus and distal trachea, avoiding the need for a tracheal tube through the incision (Figure $1, B$ ). ${ }^{11}$

Our preferred method of reconstruction is to start with a double-armed running suture on the lateral wall of the trachea to the left main bronchus in a parachute technique. Then, we place an additional interrupted suture in the anterior portion to tie the anterior thread of the running suture and secure the anastomosis. With the posterior thread of the running suture, we complete the anastomosis of the membranous part of the trachea and left main bronchus and secure the tie with an additional interrupted suture. Then, the right main bronchus (in the case of no lung resections) or bronchus intermedius is reimplanted to this new joint with a double-armed running $360^{\circ}$ suture, following a similar technique as in a right upper sleeve anastomosis (Video 1). It is recommended to cover the anastomosis at the end using a thymus flap (being careful of maintaining the blood supply).

These procedures can also be performed under spontaneous ventilation (tubeless VATS). Avoiding the endobronchial tube makes the anastomosis easier, but tubeless VATS procedures should only be performed in centers with extensive experience in nonintubated techniques. ${ }^{12}$

The rapid development of uniportal VATS in just one decade only has allowed us to evolve from simple lobectomies to accomplish the most challenging bronchovascular and carinal reconstructions in expert hands. The future of this journey is promising, and technology will help thoracic surgeons to develop even less-invasive approaches for these complex surgeries.

\section{Conflict of Interest Statement}

The authors reported no conflicts of interest.

The Journal policy requires editors and reviewers to disclose conflicts of interest and to decline handling or reviewing manuscripts for which they may have a conflict of interest. The editors and reviewers of this article have no conflicts of interest.

\section{References}

1. Chen H, Huang L, Xu G, Zheng B, Zheng W, Zhu Y, et al. Modified bronchial anastomosis in video-assisted thoracoscopic sleeve lobectomy: a report of 32 cases. J Thorac Dis. 2016;8:2233-40.

2. Zhou S, Pei G, Han Y, Yu D, Song X, Li Y, et al. Sleeve lobectomy by videoassisted thoracic surgery versus thoracotomy for non-small cell lung cancer. $J$ Cardiothorac Surg. 2015;10:116.

3. Ng CS. Thoracoscopic sleeve resection-the better approach? J Thorac Dis. 2014;6:1164-6.

4. Soultanis KM, Chen Chao M, Chen J, Wu L, Yang C, Gonzalez-Rivas D, et al. Technique and outcomes of 79 consecutive uniportal video-assisted sleeve lobectomies. Eur J Cardiothorac Surg. 2019;56:876-82.

5. Gonzalez-Rivas D, Fernandez R, Fieira E, Rellan L. Uniportal video-assisted thoracoscopic bronchial sleeve lobectomy: first report. J Thorac Cardiovasc Surg. 2013;145:1676-7.

6. Gonzalez-Rivas D, Yang Y, Stupnik T, Sekhniaidze D, Fernandez R, Velasco C, et al. Uniportal video-assisted thoracoscopic bronchovascular, tracheal and carinal sleeve resections. Eur J Cardiothorac Surg. 2016;49:16-16.

7. Soultanis KM, Gonzalez-Rivas D. Uniportal video-assisted sleeve resections: how to deal with specific challenges. J Thorac Dis. 2019;11(suppl 13): S1670-7.

8. Gonzalez-Rivas D, Delgado M, Fieira E, Mendez L. Single-port video-assisted thoracoscopic lobectomy with pulmonary artery reconstruction. Interact Cardiovasc Thorac Surg. 2013;17:889-91.

9. Gonzalez-Rivas D, Delgado M, Fieira E, Fernandez R. Double sleeve uniportal video-assisted thoracoscopic lobectomy for non-small cell lung cancer. Ann Cardiothorac Surg. 2014;3:E2.

10. Huang J, Li J, Qiu Y, Xu X, Sekhniaidze D, Chen H, et al. Thoracoscopic double sleeve lobectomy in 13 patients: a series report from multi-centers. J Thorac Dis. 2015;7:834-42.

11. Lyscov A, Obukhova T, Ryabova V, Sekhniaidze D, Zuiev V, GonzalezRivas D. Double-sleeve and carinal resections using the uniportal VATS technique: a single centre experience. J Thorac Dis. 2016;8(suppl 3): S235-41.

12. Jiang L, Liu J, Gonzalez-Rivas D, Shargall Y, Kolb M, Shao W, et al. Thoracoscopic surgery for tracheal and carinal resection and reconstruction under spontaneous ventilation. J Thorac Cardiovasc Surg. 2018;155:2746-54. 